\title{
Research on Mobile Learning Based on Augmented Reality
}

\author{
Shen Zheng \\ Quanzhou Normal University, Quanzhou, China \\ Email: 492914016@qq.com
}

Received 24 November 2015; accepted 26 December 2015; published 29 December 2015

Copyright (C) 2015 by author and Scientific Research Publishing Inc.

This work is licensed under the Creative Commons Attribution International License (CC BY).

http://creativecommons.org/licenses/by/4.0/

(c) (i) Open Access

\begin{abstract}
Augmented reality, referred to as AR, is a new technology that develops on the basis of virtual reality. It can be applied in mobile learning to create a real learning environment, which will greatly improve the efficiency and effectiveness of learning. This paper discusses the basic characteristics of augmented reality and introduces a mobile learning tool based on it.
\end{abstract}

Keywords

Augmented Reality, Mobile Learning, Basic Characteristics, Development Trend

\section{Introduction}

Augmented reality, referred to as AR, is a new technology that develops on the basis of virtual reality. It integrates computer-generated scenes into the real world, expands and complements the real world rather than completely replaces the real world, thereby strengthening user's sensory and cognitive reality. Highly popular, portable and easy to use hand-held devices, such as smart phones, which achieve the miniaturization and portability of the augmented reality system and are one of the best platforms that embody the value of augmented reality, will greatly expand and deepen the scope and depth of the use of augmented reality system.

Mobile learning is a new form of learning, and learning content of it is fragmented, short and suitable for the mobile media. Augmented reality is authentic, interactive and real-time and can be applied in mobile learning to render better learning content, to create a real learning environment and to provide high-quality learning experience, which will greatly improve the efficiency and effectiveness of learning. In this paper, based on the discussion of the basic characteristics of augmented reality, a mobile learning tool based on augmented reality is introduced.

\section{The Basic Characteristics of Augmented Reality}

Augmented reality integrates the synthetic sensory information into the user's perception of the real world [1].

How to cite this paper: Zheng, S. (2015) Research on Mobile Learning Based on Augmented Reality. Open Journal of Social Sciences, 3, 179-182. http://dx.doi.org/10.4236/jss.2015.312019 
The target of augmented reality system is to integrate the interactive real world with interactive computer-generated world, in such a way that they appear to be an environment. Augmented reality has two characteristics: the notion that video environment in digital space just is the real environment; technically, through the seamless integration of image and three-dimensional geometric model to achieve virtual-real fusion. Augmented reality technology is mainly composed of the following aspects.

1) Display system

Display system mainly provides the acquisition and display capability of intelligent terminal, including display screen, camera, is an important device for enhancing the applications of augmented reality. The current display screen of smart terminal is more than 3.5-inch, color and resolution of display screen increase gradually. Camera Resolution usually reaches one million level to ten million level, you can shoot high-definition images.

2) Interactive system

Interactive system is the primary means to influence the experience of augmented reality. Currently, smart phones use touch screen interaction, voice interaction and other interactions, to have a great change on human-machine relationship, the interactive reaction can be carried out via multi-channel with the virtual information generated by computer, allowing users go into the scene more naturally, with more fresh sense of the experience.

3) Communication system

Wireless communication technology and Internet technology are mobile services supporting technology. In some applications of augmented reality, large amounts of data is stored through a remote server, some of the data processing must be done by a remote server. $3 \mathrm{G}$ and other wireless communications technology enhance the development of augmented reality's applications by providing network bandwidth and other supports.

4) Three-dimensional graphics rendering

The algorithm of modeling and rendering three-dimensional graphics superimposes three-dimensional objects which can enhance the display in a real environment, to help users understand the environment.

5) Target recognition

Target recognition refers to fine a given target object in the relevant scene, and mark it. Augmented reality needs to implement real-time object recognition in complex moving scenes, such as feature extraction and recognition of complex scenes using multi aspects such as color, texture and contour [2]. Augmented reality system through analysis of large amounts of location data and scene information to ensure that computer-generated virtual objects can be accurately positioned in a real scene, which typically includes three basic steps: a) get and analyze scene information; b) generate virtual image; c) combine the actual situation with image stream to generate fusion scene, shown in Figure 1.

\section{A Mobile Learning Tool Based on Augmented Reality}

With the smart phone's powerful use, we have adopted a learning tool based on augmented reality in the teaching. This learning tool has the following features.

1) Superposition of virtual information and real scenery. Information point is the basic unit of data, including location information and explanatory material. Webcams can be displayed in the camera screen of the mobile device, and overlaying the icon information and orientation information. Learners can determine the location of information points by the screen's indication, and then along the direction to find information points.

2) Multi-view display of information points. In addition to the camera interface, the learning tool provides list interface and map interface. Learners can view information points or learning content by any view. Knowledge about information points, including text, pictures, video clips and the like, can be provided for learners.

3) Create new content. Learners can use the phone to create a new information point, shoot pictures, add text, and automatically record the geographic coordinate, submit to the server. These new resources can be open to other users sharing the platform.

The basic process throughout the software is shown in Figure 2.

The software is a mobile application running on phone or tablet. According to the development practices of Android application, the software structure is divided into UI layer, scheduling layer and application service layer, the layers are responsible for the following functions.

1) UI layer is mainly composed by a number of Activities and Views, among them, the main display interfaces have CameraView, MapView, ListView, WebView and SignView. 


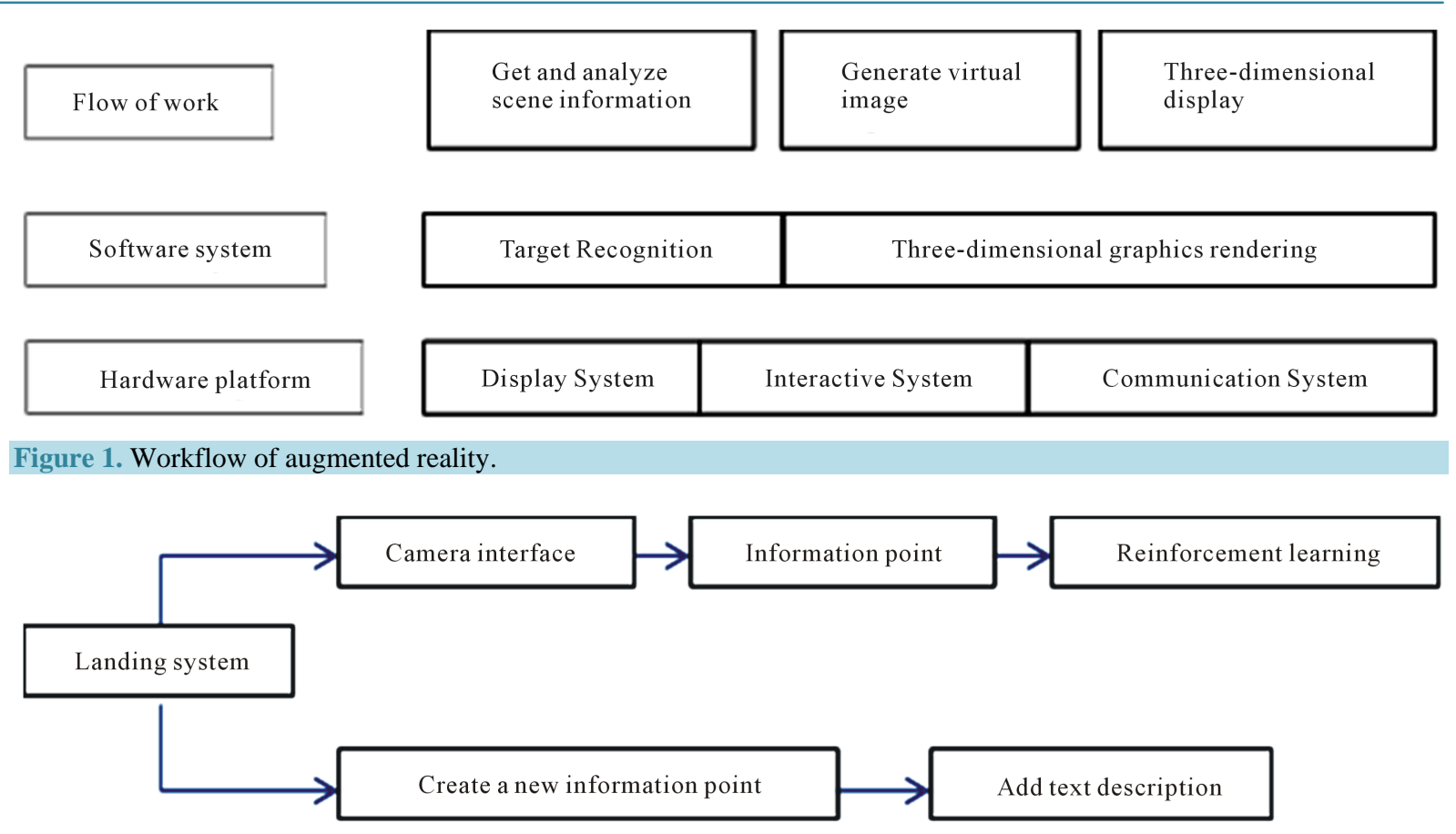

Figure 2. Software process.

2) Scheduling layer is divided into the service level management module, service binding module, message forwarding module and exception notification modules, mainly to complete the interaction of UI layer and backoffice services, including messaging, exception handling.

3) Application service layer is composed by Service, Receiver and Content-Provider, is responsible for communicating with the system, monitoring and dealing with system behavior, and messaging with the scheduling layer, including reading and writing data, accessing the content of the information point and updating location information.

\section{The Development Trend of Educational Applications of Augmented Reality}

For its characteristics as the virtual-real fusion, the real-time interaction and three-dimensional immersion, augmented reality environment has a new difference with the learning mode based on traditional learning management platform and the three-dimensional virtual learning environment like Second Life and Sloode [3]. Especially its 3D immersive environment offers a new possibility on teaching object modeling, teaching experience, the presentation of teaching result and the interaction of teachers and students. Through the introduction of augmented reality, we have analysis of the following points about the development trend of augmented reality mobile learning.

1) Expand the teaching content and teaching activities

When students and teachers are faced with not a flat or pure three-dimensional space, but a learning environment that integrated by virtual objects and real scene, just like augmented reality, the traditional means of interaction may not adapt. But it also provides more space can be explored, such as how to establish course content by situated cognition theory and learning activities theory in the environment of virtual-real fusion, how to create teaching activities, how the communication between learners be more direct, how to build users' learning experience model and interactive behavior model, all these problems wait the developers and users of augmented reality learning environment to explore.

2) Integration with existing learning management systems

As a nascent technology, augmented reality environment must be able to share data with existing information systems, or have some kind of association, in order to be accepted by mainstream educators. But to put together two distinct environments, we need more people to use it and the intensive study of how this integration environment to enhance teaching effectiveness, so as to comply with existing and new teaching methods. 
3) Integration with intelligent technology

The current augmented reality learning environment is only able to create three-dimensional graphics and simple interaction, but learning is a very complex activity process, the ideal augmented reality learning environment should be able to mimic the experience, methods and behavior of teaching instructors, and have more friendly interactive methods.

\section{Conclusion}

With the popularity of mobile learning technology, more and more people will choose this way of learning. And the usability and interactivity make augmented reality technology have unique advantages in terms of building real situation. Augmented reality will become an important part of mobile learning.

\section{References}

[1] Vallino, J.R. (1998) Interactive Augmented Reality. University of Rochester, New York.

[2] Yao, Y. (2006) The Application Research of Augmented Reality. Zhejiang University, Hangzhou.

[3] Cai, S. and Yu, S.Q. (2010) Sloodle: A Case for 3D Virtual Learning Environment. Open Education Research, 16, 98104. 